\title{
MIGRACIÓN DE LO PRESENCIAL A LO VIRTUAL EN LA ASIGNATURA INTRODUCCIÓN A LA COMPUTACIÓN DEL PROGRAMA DE ENFERMERÍA DE LA UCLA
}

\author{
(MIGRATION OF THE ACTUAL THING TO VIRTUAL IN THE SUBJECT "INTRODUCTION TO \\ COMPUTERS” IN NURSING PROGRAM AT UCLA)
}

\author{
Graciela Henríquez Gabante \\ Eunice Ugel Garrido \\ Universidad Centroccidental Lisandro Alvarado, UCLA (Venezuela)
}

\section{RESUMEN}

El Decanato de Ciencias de la Salud de la Universidad Centroccidental Lisandro Alvarado está desarrollando un proceso de migración de lo presencial hacia lo virtual en los programas de Medicina y Enfermería. Los docentes de la asignatura Introducción a la Computación están trabajando en el cambio curricular a la virtualidad, el cual se ha producido en 4 fases: capacitación docente para gestionar asignaturas en línea, rediseño del programa, selección de medios y material, y montaje del curso en la plataforma Moodle como entorno virtual de aprendizaje. Las tres primeras fases ya están culminadas, la cuarta aún está en proceso de ejecución. De esta experiencia se concluye que la capacitación fue bien percibida por los docentes, quienes asumieron el reto de emigrar al e-learning, pero la carga académica les impiden agilizar este cambio. Los equipos tecnológicos del decanato son obsoletos a las necesidades de los docentes para ejecutar sus cursos virtuales.

Palabras clave: migración, docentes, cursos en línea, plataforma Moodle.

\begin{abstract}
The dean of health sciences at Lisandro Alvarado Centroccidental University is currently developing a system in which the traditional onsite programs for medicine and nursing are being migrated to the virtual system. Professors from the course "An introduction to Computing" are also working on making curricular changes to adapt to the new system. This is being done in four stages: 1) teacher training- to manage online subjects; 2) course design changes; 3) the selection of technological tools and materials and 4) the uploading of the course to the Moodle platform, which is the virtual learning environment. The first three phases have already been completed, but the fourth stage is currently being implemented.
\end{abstract}


As a result, the training received positive feedback by professors, i.e. those who took on the challenge of migrating to e-learning. However, the burden of academic work impeded the smooth transition to making this change. The technological equipment that was being used by the university governors was therefore not not fully modernized to satisfy the needs of profesors or to effectively deliver online courses.

Keywords: technological migration, professors, online courses, the Moodle platform.

En esta sociedad del conocimiento la ciencia y la tecnología han impregnado los distintos ámbitos de la vida humana. Han cambios los modos de pensar, sentir y actuar, influenciados por el creciente contenido técnico y cada vez mayor ocupaciones de alta tecnología (Cardona, 2002). Además, se ha generado una nueva forma de vivir reduciendo la concepción del tiempo y del espacio. Hoy es una realidad el telecomercio, telebanca, teletrabajo, teleformación, telemedicina, teleenseñanza, entre otras, actividades que se realizan de forma sincrónica o asincrónica sin barreas espacio temporales.

El sector educativo está inmerso en esta realidad. La UNESCO (1998) en la conferencia mundial sobre la educación superior, uno de los aspectos que resalta es el potencial y desafío de las tecnologías, y los métodos educativos innovadores para lograr las universidades del siglo XXI, en lo que se destaca: a) la educación permanente, b) sin fronteras ni barreras, c) centrado en el aprendiz, d) basada en la informática y la telemática, e) enseñanza y aprendizaje de manera colaborativa y colectiva a través de las redes sociales de Internet, f) con relativa independencia, y g) mediante representaciones electrónicas digitales de objetos, contenidos, procesos y personas.

En este mismo orden de ideas, en la Conferencia Regional de Educación Superior (CRES, 2008) en el informe Tendencias de la Educación Superior en América Latina y el Caribe, en el apartado Reforma de la Educación Superior, se indica la transcendencia de la formación superior en el siglo XXI, ante los imperativos de la sociedad del conocimiento, destacando el avance en el empleo de tecnologías de información y comunicación digitales. Es así como una de las tendencias de la educación superior es el asentamiento y desarrollo de la universidad virtual a través de los medios tecnológicos, especialmente la Web 2.0.

Sin embargo, las universidades presenciales tradicionales deben prepararse para alcanzar este cometido, dado que sus características son usualmente opuestas a las de las universidades del XXI propuesta por la UNESCO y CRES. Estas universidades tradicionales en su mayoría se caracterizan por tener una infraestructura, recursos 
y profesores bajo un paradigma educativo rígido. Generalmente, en el proceso de enseñanza y aprendizaje el centro de preocupación está en el que enseña y no en el que aprende, el profesor es un transmisor de la información y el uso de la tecnología de información y comunicación (TIC) es relativamente escaso.

En este sentido, para estas universidades tradicionales no es fácil cambiar a un nuevo paradigma de aplicación tecnología en el proceso educativo. De allí que la integración de las TIC en las aulas es compleja, se genera a largo plazo. La UNESCO (2008) sugirió que uno de los primeros pasos para incorporar las TIC en el proceso educativo, es invertir en capacitación, en lograr que la planta profesoral tenga las competencias básicas en el uso tecnológico, así como en su fundamentación pedagógica. Ello debido a que gran parte de la responsabilidad de su buen uso recae sobre el profesor, el cual tiene un papel determinante en el proceso de enseñanzaaprendizaje, es un mediador entre el currículo y sus estudiantes. Esta capacitación tecnológica y pedagógica permitirá a los docentes poner en práctica acciones innovadoras que mejoren el ambiente del aula. A su vez los ayudará a sentirse impulsados a asumir el nuevo rol al que están llamados de acuerdo a las exigencias tecnológicas de esta sociedad del conocimiento.

En este contexto, en Venezuela, la Oficina de Planificación del Sector Universitario (OPSU) está trabajando en el Proyecto Nacional de Educación Superior en el cual se está incorporando la educación a distancia $(\mathrm{EaD})$ mediada por la tecnología, para ello ha propuesto a las universidades tradicionales venezolanas la incorporación de las TIC en sus procesos educativos (Ministerio del poder popular para la educación universitaria, s/f). La coordinadora del proyecto de $\mathrm{EaD}$ a nivel nacional expresó se que está trabajando en la normativas y reglamentos, ya que no existen en el país antecedentes sobre la generación y ejecución de normativas nacionales para el diseño, desarrollo y evaluación de sistemas de estudios a distancia (AVED, 2008). La Universidad Centroccidental Lisandro Alvarado (UCLA) juntos con otras universidades del país están trabajando en este proyecto nacional de EaD.

Respecto a la UCLA, es una institución educativa venezolana de carácter público que ofrece 18 carreras de las áreas científicas, tecnológicas y humanísticas, bajo la modalidad de educación presencial, cuenta con siete Decanatos: Agronomía, Ciencias Veterinarias, Administración, Ciencia y Tecnología, Humanidades y Artes, Ingeniería Civil, y Ciencias de la Salud. La UCLA articulada con lo planteado por la UNESCO, CRES y OPSU, ha creado la instancia Sistema de Educación a Distancia (SEDUCLA), desde el año 2001 viene desarrollando un proceso de migración de lo 
presencial hacia lo virtual hasta lograr implementar la educación a distancia mediada por la tecnología en las carreras que oferta la institución (Pérez, 2008).

Con relación al Decanato de Ciencias de la Salud (DCS), en lo últimos lapsos académicos viene presentando un crecimiento exponencial dela matricula estudiantil, utilizando los mismos recursos y un presupuesto deficiente para atender la demanda estudiantil. De modo que este decanato está en la necesidad de emigrar los diseños curriculares de los programas de Medicina y Enfermería bajo la modalidad a distancia. Esta migración permitirá solventar algunos de los problemas que presenta el DCS, entre lo que se destaca: a) escaso espacio físico, existen pocas aulas para formar grupos reducidos de estudiantes para impartir el proceso educativo centrado en el aprendiz, usualmente los docentes se ven obligados a impartir clases magistrales para atender a todos los estudiantes simultáneamente, convirtiéndose el profesor es un transmisor de la información; b) contratación de nuevo personal, el presupuesto es insuficiente para contratar profesores que atiendan la demanda estudiantil, un docente usualmente atiene a la totalidad de estudiantes inscritos; c) accesibilidad a la institución, dificulta de aproximadamente más del $15 \%$ de los estudiantes en trasladarse hasta la institución, vive geográficamente distante del decanato. Razones por las cuales el Cuerpo Directivo del DCS con el apoyo de SEDUCLA se ha propuesto como meta a largo plazo ofertar los programas de Medicina y Enfermería bajo la modalidad presencial y a distancia.

En este sentido, SEDUCLA-Cs de la Salud para iniciar la propuesta de incorporar la $\mathrm{EaD}$ en los diseños curriculares de los programa de Medicina y Enfermería, diagnosticó las competencias en el uso de las TIC que poseen los docentes del decanato. Como resultado se obtuvo: a) 80\% de los docentes son especialistas en el área de la salud y no necesariamente usuarios asiduos de las tecnologías; b) 35\% de los docentes resultaron adeptos a las TIC quienes pueden cometer el error de orientar su proceso educativo hacia la tecnología y no profundizar en el diseño pedagógico. Por otra parte, $30 \%$ se opone a la implementación de la EaD mediada por las tecnologías, bien sea por resistencia al cambio, apego a lo tradicional, miedo a lo desconocido o falta de capacitación; c) en cuanto al uso de las TIC, los resultados indicaron que más del 70\% posee las competencias en los programas ofimática siendo el de mayor uso el procesador de palabras Word, seguidamente de PowerPoint y el menos utilizado es el Excel; d) más del 90\% utilizan solamente el Windows desconociendo otros sistemas operativos Linux, UNIX, entre otros; e) en cuanto a las herramientas de Internet, el correo electrónico es el más utilizado, teniendo dificultad en usar otras herramientas de Internet. Es importante resaltar que 80\% los docentes expresaron 
no tener las competencias en las plataformas educativas Moodle, e incluso hubo quienes expresaron no saber que era Moodle (Henríquez, 2008).

Realizado este diagnóstico, en octubre del 2008 se inició el primer plan de capacitación, ofertando dos cursos: Curso Computación para nivelar a los docentes con poca experticia en el uso del Microsoft Office e Internet, y el Diplomado de Entornos Virtuales de Aprendizaje (EVA). Este diplomado tuvo como propósito capacitar a los docentes del DCS como facilitadores, tutores y diseñadores de cursos en línea, así como también, orientarlos en la planificación de la instrucción mediante el modelo instruccional ASSURE, permitiéndoles de esta manera diseñar, ejecutar, administrar y evaluar cursos virtuales bajo la plataforma Moodle.

Este diplomado EVA fue el punto de partida para que los docentes de la asignatura Introducción a la Computación (IC) del programa de Enfermería comenzaran a trabajar en el cambio curricular de lo presencial a lo virtual. El propósito de la asignatura es que el futuro profesional adquiera las competencias en el manejo del computador, especialmente en Windows, el Microsoft Office Word y PowerPoint y el paquete estadístico SPSS. El desarrollo de las clases es presencial utilizando la metodología aprender haciendo, donde después de la exposición del docente los estudiantes ejercitan lo aprendido. Desde el lapso académico II-2002 ha funcionado con los mismos recursos: dos docentes, dos auxiliares docentes y un laboratorio de computación con 20 computadoras. Cada lapso académico la matricula estudiantil tiende a incrementar, teniendo que dividir en diferentes horarios el total de inscritos en grupos de 20 estudiantes máximo. Aproximadamente el 25\% de los estudiantes abandonan la asignatura, por no poder cumplir con el horario del grupo donde quedan asignados. Es por ello, que esta asignatura se ve en la necesidad inminente de ofertarla bajo la modalidad presencial y a distancia. De allí que el presente trabajo tiene como objetivo describir la experiencia del proceso de migración de lo presencial a lo virtual en la asignatura IC.

\section{MARCO TEÓRICO}

El siglo XXI está caracterizado por la sociedad del conocimiento, donde la información y el conocimiento se han convertido en los activos cruciales para la producción, la competitividad, el crecimiento y desarrollo económico. Siemens (2007) señaló que en esta era del conocimiento, el desarrollo y divulgación de la información, son prácticamente instantáneos, la mitad de lo que es conocido hoy no lo era hace 10 años, el conocimiento crece exponencialmente. Por tanto, las TIC 
se convierten en elemento de impulso y desarrollo económico, político y social, abriendo camino a un mundo globalizado, dinámico y cambiante.

Otro factor que caracteriza a esta sociedad del conocimiento es la posibilidad de obtener todo tipo de información, sobre cualquier asunto, presentada en diferentes formatos (texto, hipertexto, gráfico, imagen, y sonido), al momento y desde cualquier lugar del mundo. En otras palabras, las telecomunicaciones han generado nuevas concepciones del tiempo y del espacio; la multimedia ha facilitado el uso del computador como herramienta de integración de medios como audio, video, sonido, imágenes, texto, animación, entre otros; y el hipertexto ha promovido nuevas formas de lectura y escritura (López, Espinoza y Flores, 2006).

Ahora bien, si el sector educativo es quien forma a los profesionales que demanda la sociedad, la cual esta siendo invadida por la revolución tecnología, se hace imperante cambiar el modelo educativo tradicional, para preparar ciudadanos competentes tecnológicamente, quienes se afrontaran a un mundo laboral sin fronteras, dinámico y cambiante. Es necesario saltar del modelo de enseñanza basado en la transmisión de información pre-digerida, a uno que fomente el sentido de la interacción, la participación activa, la resolución de problemas, la flexibilidad, el espíritu crítico, la responsabilidad, el aprender-aprender, entre otras (Silvio, 2004).

Es así como las TIC traen consigo un nuevo paradigma a la educación, conduciendo los procesos institucionales para que incorporen el rol mediador de los recursos tecnológicos, donde la dinámica del aprendizaje no se centra solamente en una asimilación pasiva de contenidos, sino que son complementadas con estrategias instruccionales que conducen hacia la construcción del aprendizaje, asumiendo en el estudiante posturas responsables y autónomas (García Aretio, 2001). Pero, es importante resaltar lo expresado por Tobón (2007), que a pesar de la autonomía con la que el estudiante trabaja en los ambientes virtuales, sigue siendo el docente quien dirige el proceso, asigna tareas, establece los objetivos, planea actividades y asigna tiempo para la realización de las mismas, es decir, es quien tiene el control sobre la instrucción.

Esta afirmación de Tobón permite corroborar que a pesar que las tecnologías están disponibles para ser utilizadas, dependerá del profesor evaluar si son útiles para sus objetivos, si pueden mejorar su trabajo y en qué medida. El uso de las TIC en el proceso educativo deberá conducir a lo que se espera que el alumno aprenda, y los resultados obtenidos reconsiderarán los objetivos y las estrategias a utilizar. Por ello, la UNESCO (2008) indicó que para utilizar pedagógicamente las TIC, es 
necesario considerar las competencias tecnológicas que tienen los docentes en las universidades tradicionales al integrarla en los procesos educativos.

En conclusión, las instituciones educativas tradicionales deben capacitar a los docentes en el uso pedagógico de las TIC para incorpóralas en el diseño de la instrucción con buen sentido didáctico. Además, la motivación de los aprendices, y el logro de los resultados de aprendizaje, fundamentalmente están asociados con el diseño instruccional que se ponga en práctica (Santos, 2007).

Se entiende por Diseño Instruccional el proceso sistemático mediante el cual se analizan las necesidades y metas de la enseñanza, se seleccionan y desarrollan las actividades y los recursos que permitan alcanzar las metas fijadas, así como los procedimientos de evaluación del aprendizaje y de toda la instrucción (Dick, Carey y Carey, 2001; Gustafson y Branch, 2002; Heinich, Molenda, Russell y Smaldino, 2002; Cookson, 2003; Tobón, 2007).

Ahora bien, para orientar la planificación de la instrucción, se conocen diversos modelos de diseño instruccional, donde muchos de ellos constan de las siguientes fases: análisis, diseño, desarrollo, implementación y evaluación. Entre lo más conocidos se encuentran el Modelo: ADDIE; Dick y Carey; Ely y Gerlach; ASSURE de Heinich et al.; Newby, Stepich, Lehman, y Russell; Morrison, Ross y Kemp.

En el plan de capacitación del DCS se ha seleccionado el Modelo ASSURE como un marco conceptual y comunicacional apropiado para guiar, dirigir, administrar y evaluar procesos instruccionales en línea de alta calidad académica. La aplicación del modelo no requiere una alta experticia de los docentes en teorías de diseño instruccional, ni se requiere de inversiones elevadas en recursos administrativos o tecnológicos.

El Modelo ASSURE es un modelo básico de diseño de la instrucción propuesto por Heinich et al. (2002) para guiar y asegurar la planificación sistemática, paso a paso, del más conocido evento instruccional: la lección. El acrónimo ASSURE significa: A - analizar a los aprendices (Analyze learners), $\mathbf{S}$ - formular los objetivos de aprendizaje (State learning objectives), $\mathbf{S}$ - seleccionar métodos, medios y materiales (Select methods media and materials), $\mathbf{U}$ - utilizar métodos medios y materiales (Utilize methods media and material), $\mathbf{R}$ - requerir la participación de los aprendices (Require learner participation) y $\mathbf{E}$ - evaluación y revision (Evaluate and revise). A continuación se describe cada una de las fases. 
Analizar a los aprendices: Se identifican las capacidades, necesidades e intereses de la audiencia. Se resalta la importancia de conocer las características generales y específicas de los participantes, tales como: edad promedio del grupo, nivel socioeconómico y cultural, habilidades o competencias de entrada, experticia en el uso de determinada tecnología, actitudes hacia el tema de estudio planteado, factores motivacionales, estilos de aprendizaje, entre otros.

Formular los objetivos de aprendizaje: En esta fase se precisa en términos de conductas observables y medibles lo que se espera que cada aprendiz adquiera y domine al final de cada experiencia de aprendizaje. En general, se pretende determinar cuáles serán sus conocimientos (saber conocer), actitudes y valores (saber ser) y desempeños (saber hacer), a lograr al culminar la experiencia de aprendizaje.

Seleccionar métodos, medios y materiales: El facilitador determina las ayudas didácticas, métodos o recursos disponibles para el desarrollo de la instrucción en función del logro de los objetivos de aprendizaje. Escoge entre métodos, medios y materiales disponibles, modifica los existentes o diseña unos nuevos, esenciales para el logro de los objetivos. Si una estrategia de enseñanza funciona bien en modalidad presencial, probablemente requiera algunos ajustes para que funcionen bien a distancia.

Utilizar métodos medios y materiales: Hacer uso adecuado de un medio, método o material, significa centrar en los aprendices el uso de los recursos para el desarrollo de sus propias actividades de aprendizaje.

Requerir la participación de los aprendices: En esta fase se precisan las actividades y estrategias de aprendizaje centradas en los aprendices que les demanden una participación activa. Se resalta la importancia de mantener a los estudiantes involucrados activamente en sus propios procesos de aprendizaje, creando intencionalmente diversas situaciones de aprendizaje que los obligue a interactuar con los contenidos, con los facilitadores, con sus compañeros de curso y con otros expertos.

Evaluación y revisión: Esta última fase permite determinar si se cumplió con los objetivos del curso, tiene como propósito: a) revisar con sentido crítico y constructivo el desarrollo de todo el proceso de instrucción para determinar sus aciertos y desaciertos; b) determinar en los aprendices su nivel de aprovechamiento o logro de los resultados previstos en los objetivos de aprendizaje o las competencias planteadas; c) evaluar la instrucción, midiendo la efectividad de sus métodos y 
recursos; d) determinar las discrepancias entre los resultados previstos y los logrados al final de la instrucción; e) determinar las deficiencias de los medios, métodos o materiales usados; y f) precisar en forma cualitativa el nivel de satisfacción alcanzado, tanto por el facilitador como por los participantes.

Para aplicar el Modelo ASUURE en el programa de la asignatura IC es imprescindible conocer su ubicación, audiencia, propósito, contenido programático, entre otras características. En este sentido, la asignatura IC está adscrita al programa Enfermería y está ubicada en el segundo semestre del pensum de estudio. Tiene como propósito que el futuro profesional adquiera las competencias en el uso del computador. Está dividida en cinco unidades: Estructura del Computador, Sistema Operativo Windows, Procesador de Palabras Microsoft Word, Presentación de Diapositivas Microsoft Power Point y Paquete Estadístico SPSS. Esta asignatura constituye una entrada a diferentes líneas curriculares del plan de estudio.

Es importante destacar que desde su creación en el lapso académico 1998-I, se ofrece solamente bajo la modalidad presencial, es a partir del lapso académico 2011-1 que se ofrecerá tanto presencial como a distancia mediada por las tecnologías. Actualmente se están realizando los cambios tanto del programa como del diseño instruccional para emigrar de lo presencial a lo virtual.

\section{DESCRIPCIÓN DE LA EXPERIENCIA}

El proceso de migración de lo presencial hacia lo virtual en la asignatura IC se ha realizado en cuatro fases, las cuales se describirán a continuación.

Primera fase: Capacitación delos docentes en el Diplomado EVA. Esta capacitación además de formar a los docentes como facilitadores y/o tutores a distancia, los orientó en el diseño instruccional mediante el modelo ASSURE, permitiéndoles de esta manera rediseñar la instrucción bajo la plataforma Moodle. Esta fase se inició en octubre del 2008 y tuvo una duración de 6 meses.

Segunda fase: Se rediseño el programa de la asignatura, el cual se inició en abril del 2009. Se utilizó el modelo ASSURE y los elementos del currículo por competencia como el saber (conocimiento), saber hacer (habilidad) y saber ser (actitud) propuesto por la comisión de currículo de la UCLA. Lo primero que se hizo fue analizar a los aprendices, conocer sus características generales y específicas, tales como: edad, nivel socioeconómico y cultural, competencias de entrada, experticia en el uso tecnológico, actitudes hacia la asignatura, factores motivacionales, estilos 
de aprendizaje, promedio del grupo, entre otras. Para ello fue necesario formularse y responder algunas preguntas que orientaron a tener una visión más especifica de los estudiantes a quienes va dirigida la instrucción, entre las que se destacan: ¿̇e tiene información sobre el nivel de instrucción previo de los aprendices?, ¿Están los aprendices suficientemente interesados y motivados para cursar la asignatura en línea?, ¿Se ha tomado alguna decisión con respecto a los estilos de aprendizaje de los aprendices en situaciones de formación en línea?, ¿Será necesario organizar algunos encuentros presenciales o toda la instrucción se puede facilitar en línea?, ¿Se ha recogido información sobre la experticia de los aprendices en el uso de correo electrónico, Internet, y determinado software o tecnología?, ¿Se tiene información precisa sobre las posibilidades de acceso de los aprendices a la instrucción entregada vía Internet? Para responder estas preguntas se diseñó un instrumento de exploración, conocida como "Prueba Exploratoria".

Conocida la audiencia, se procedió a formular los objetivos de aprendizaje. En esta fase se enunció en términos de conductas observables y medibles lo que se espera que cada aprendiz adquiera y domine al final de cada experiencia de aprendizaje. En general, se determinó cuáles serán los conocimientos (saber conocer), actitudes y valores (saber ser) y desempeños (saber hacer), a lograr al culminar la experiencia de aprendizaje. Básicamente, la formulación precisa y correcta de los objetivos de aprendizaje debería evitar que la instrucción y la evaluación vayan por sentidos opuestos. Algunas preguntas que orientaron esta etapa fueron: ¿Están los objetivos formulados en términos de lo que los aprendices deben saber conocer, saber hacer o saber ser? ¿Se han formulado objetivos que estimulen altos procesos cognitivos? ¿Son específicos los resultados de aprendizaje previstos en los objetivos? ¿Se han formulado los objetivos con el uso de verbos que señalan inequívocamente conductas observables y medibles? ¿Los objetivos están formulados de manera que ayuden al estudiante a pasar progresiva y crecientemente por los diferentes niveles cognitivos, actitudinales y psicomotrices? Finalmente, se analizaron las estrategias de aprendizaje, recursos y evaluaciones de los aprendices en función de los objetivos, logrando terminar en noviembre el 2009.

Tercera fase: Se elaboraron las guías didactas y se seleccionaron los medios y materiales a utilizar, relacionada al área de la Salud principalmente con el profesional de enfermería. Entre las preguntas que orientaron esta etapa se destacan: ¿Qué tipo de materiales de apoyo pueden emplearse para facilitar diversas situaciones de aprendizaje que demandan la participación activa, reflexiva y analítica de los aprendices?,¿¿Quétipo de experiencias pueden emplearseparafacilitarla comprensión global de los temas estudiados?, ¿Existen problemas, proyectos o estudios de casos 
a ser propuestos? Si van a utilizarse materiales previamente concebidos para la educación presencial ¿se han ajustado a la modalidad de educación a distancia?

Esta fase se inició en noviembre del 2009, aunque se da por concluida, actualmente sigue en ejecución, ello en virtud que en Internet existen guías, presentaciones, videos y otros recursos, con información acorde al contenido programático de la asignatura los cuales se actualizan frecuentemente. Esta es la fase que más tiempo de dedicación ha requerido. A la fecha, febrero del 2011 todavía se les están haciendo ajuste a las guías didácticas y se sigue la búsqueda de información por Internet.

Cuarta fase: Diseño y montaje del curso en la plataforma Moodle. Teniendo disponible los materiales de apoyo o recursos didácticos a utilizar, así como también las evaluaciones que demuestren el logro de aprendizaje, se comenzó el montaje del curso en la plataforma Moodle. Esta fase se inició en octubre del 2010, a febrero del 2011 aun se encuentra en construcción y prueba. Solamente se ha montada la unidad I y II de la asignatura. Es importante destacar, que son los mismos docentes quienes lo están montando y tienen otras actividades que cumplir: preparar e impartir sus clases bajo la modalidad presencial, ser tutores de trabajos de investigación, cumplir con las funciones de investigación, extensión y gestión, entre otras. Se estima terminar la construcción para finales del segundo trimestre del año 2011 y poder poner a prueba la ejecución para el lapso académico 2011-1, el cual se tiene programado comenzar en julio del 2011. Se estima que la puesta en marcha de la EaD en la asignatura IC responda a los problemas que presenta la mencionada asignatura.

\section{RESULTADOS}

El proceso de migración de lo presencial a lo virtual en la asignatura IC ha dado como resultado:

- Cuatro docentes capacitados con competencias para asumir el rol de facilitador y/o tutor para impartir cursos a distancia, así como también, como diseñadores en la plataforma Moodle. Es importante mencionar que la capacitación en el Diplomado EVA fue bien percibida por los docentes, ya que los orientó sobre los fundamentos de la EaD, el rol de tutor a distancia, la importancia de diseño instruccional para la gestión de cursos en línea a través del modela ASSURE y el haber aprendido la plataforma Moodle. Esta capacitación sirvió de base en el rediseño de la instrucción de la asignatura IC mediante el modelo ASSURE y el montaje del curso en la plataforma Moodle. Cuando el curso esté en la fase de ejecución, se espera poner en práctica el rol del tutor a distancia. 
- Información general y específica de los estudiantes de la asignatura IC a través de la prueba exploratoria. En cuanto a las características genéricas se determinó que son estudiantes de enfermería, con edad promedio de 20 años, nivel socioeconómico de medio a bajo y condición académica regulas. Entre las características específicas se destacan:

- Conocimientos previos en el uso del computador. Del total de estudiantes, $31 \%$ expresó saber usar el computador, $45 \%$ sabe poco y lo que han aprendido ha sido por ensayo y error, también opinaron que lo que más usan es Internet, y de este el correo electrónico y el facebook. Es importante tener en cuanta que existe un $24 \%$ que opinó no saber nada, e incluso tienen temor en usar el computador.

- En cuanto a las competencias en los programas ofimática, del total de estudiantes, 66\% expreso que realiza manuscritos con el Microsoft Word, aunque no lo dominan del todo, y lo han aprendido por ensayo y error. Seguidamente, 29\% manifiesto que realiza presentaciones con el Microsoft PowerPoint. El que menos usan es la hoja de cálculo Microsoft Excel, solamente el 5\% manifestó conocer las opciones básicas del Excel.

- En cuanto a las competencias en el paquete estadístico SPSS, solamente el 8\% de los estudiantes tienen las competencias, el resto nunca lo ha utilizado.

- El 100\% de de los estudiantes manifestaron no conocer la plataforma educativa Moodle.

- Respecto a la disposición para cursar la asignatura a distancia mediada a través de la plataforma Moodle, a pesar de no conocer dicha plataforma, 85\% expresó estar motivado e interesado en cursar la asignatura en línea, pero con asesorías presenciales si fuera el caso, y con un primer encuentro presencial para familiarizarse con la plataforma Moodle y explicar los lineamientos de cómo se desarrollará la instrucción a distancia. EL 15\% opino que no quiere ver la asignatura a distancia ya que no tiene computadoras en su casa.

- El 25\% de los participantes no tiene computador en su casa, $20 \%$ tiene computador pero sin acceso a Internet, este grupo manifestó que si quieren participar en esta experiencia, pero les preocupa el cómo lo pueden hacer ya que les saldría muy costoso ir frecuentemente a un ciber café. El resto, $55 \%$, manifestó que si tienen computador en su casa con acceso a Internet 
y consideran que en la universidad ha sido muy lento el proceso se ofertar asignaturas en línea, más aun en este tipo de carreras, donde el estudiantes de enfermería desde los primeros semestres tienen que cumplir practicas en los centros de salud, y las asignaturas del área básica vista bajo la modalidad a distancia, les brindaría la flexibilidad horaria al no asistir presencialmente a las clases magistrales en el horario convenido, sino instruirse ajustándose a su ritmo y disponibilidad.

- Programa de la asignatura reestructuradoy adaptado al currículo por competencia propuesto por la UCLA. Los objetivos de aprendizaje y las estrategias a utilizar fueron modificados con base en los componentes: conocimientos, destrezas y actitudes. En cada una de las unidades se utilizó el siguiente formato:

\begin{tabular}{|l|l|}
\hline \multicolumn{2}{|l|}{ UNIDAD No. : } \\
\hline Duración. & Ponderación: \\
\hline
\end{tabular}

OBJETIVO TERMINAL: Finalizada la unidad el o la estudiante

\begin{tabular}{|c|l|l|l|l|l|l|}
\hline \multirow{2}{*}{$\begin{array}{c}\text { ELEMENTOS DE } \\
\text { COMPETENCIA }\end{array}$} & \multicolumn{3}{|l|}{ PLANTEAMIENTO GENERAL DE SABERES } & \multicolumn{2}{l|}{ ESTRATEGIAS } & \multirow{2}{*}{ RECURSOS } \\
\cline { 2 - 7 } & Conceptual & Procedimental & Actitudinal & Aprendizaje & Enseñanza & RECU \\
\hline $\begin{array}{c}\text { Al finalizar la unidad } \\
\text { el estudiante } 0 \text { la } \\
\text { estudiante: }\end{array}$ & & & & & & \\
\hline
\end{tabular}

- Cinco guías didácticas, una por cada unidad de estudio, con hipertexto: texto, imágenes, hipervínculos, etc; para lograr la atención del estudiante. También se han elaborado cuatro videos: 1) recomendaciones para el estudio independiente, 2) recomendaciones para uso del computador, 3) el computador y sus partes y 4) ambiente Windows.

- Curso montada en Moodle para ser ofertada a distancia. Esta fase aun está en construcción, solamente se ha ensamblado la unidad I: Conceptos Generales del Computador, y la Unidad II: Ambiente Windows. Faltando por montar las unidades III, IV y V: Microsoft Word, Microsoft Power Point y SPSS.

\section{CONCLUSIONES Y RECOMENDACIONES}

Esta experiencia de virtualizar la asignatura IC ha sido una tarea ardua, por el paradigma rígido y la falta de recursos de la institución para asumir el cambio de la modalidad presencial a la virtual. Por lo que se concluye y recomienda: 
- La capacitación del Diplomado EVA fue bien percibida por los docentes, ya que los orientó sobre los fundamentos de la $\mathrm{EaD}$, el rol de tutor a distancia, la importancia de diseño instruccional para la gestión de cursos en línea y el haber aprendido la plataforma Moodle. Se recomienda capacitar de igual forma a los estudiantes quienes también asumirán un nuevo rol.

- Los docentes de IC están motivados y comprometidos para asumir el reto de emigrar de lo presencial a lo virtual. Sin embargo, la carga académica le permite dedicar solamente 4 horas semanales a este proceso. Se recomienda a los responsables del proyecto y/o autoridades de la institución, asignar recursos humanos, bien sea para descargar a los docentes de varios de sus compromisos o asignarles personal especialistas en Moodle, diseño grafico, etc., para que ayuden a los docentes en el diseño de las guías didácticas, búsqueda de imágenes acorde al contenido de estudio, elaboración de videos, montaje del curso en línea en la plataforma Moodle, entre otras.

- Los equipos tecnológicos del DCS: computadoras, modem, equipo de video, puntos de red, entre otros, son obsoletos. Se recomienda actualizarlos o adquirir equipos acordes a las necesidades de los docentes para ejecutar sus cursos virtuales.

- Aun cuando se está diseñado esta propuesta para ofertar la asignatura a distancia, las características de los aprendices conlleva a seguirla ofertando en ambas modalidades.

\section{REFERENCIAS BIBLIOGRÁFICAS}

Cardona, G. (2002). Tendencias educativas para el siglo XXI. Educacion virtual, online y @learning. Elementos para la discusión. Edutec. Revista Electrónica de Tecnología Educativa (15).

Conferencia Regional de Educación Superior (CRES) (2008). Tendencias de la Educación Superior de América Latina y el Caribe. [en línea] Disponible en: http://www.iesalc.unesco.org.ve/index. php?option $=$ com content $\& v i e w=a r$ ticle\&id $=365 \&$ Itemid $=423$ \&lang $=\mathrm{es}$ (consulta 2011, 20 de febrero).
Cookson, P. (2003). Elementos de diseño instruccional para el aprendizaje significativo en la educación a distancia. [en línea] Disponible en: http://meidi. ula.ve/dinstruccional/archivos/ elementos.doc (consulta 2008, 10 de noviembre).

Dick, W.; Carey, L.; Carey, J. (2001). The systematic design of instruction. New York: Addison - Wesley Educational Publisher.

Dorrego, E. (2008). Proyecto Nacional de Educación Superior a Distancia. Conferencia Internacional de la 
Asociación Venezolana de Educación a Distancia (AVED). [en línea] Disponible en: $\quad$ http://www.universia.tv/ES/ VIDEO/182022 ELENA-DORREGOAVED-2008TERCERA-PARTE-FLV (consulta 2010, 17 de febrero).

García Aretio, L. (2001). La educación a distancia: De la teoría a la práctica. Barcelona, España: Ariel.

Gustafson, K.; Branch, R. (2002). Survey of instructional developments models. Syracuse, NY: ERIC Clearinghouse on Information \& Technology.

Heinich, R.; Molenda, M.; Russell, J.; Smaldino, S. (2002). Instructional media and technology learning. Upper Saddle River, NJ: Pearson Education.

Henríquez, G. (2008). Diagnóstico de competencias básicas en las TIC de los docentes del decanato de ciencias de la salud. XIV Congreso Internacional de Tecnología y Educación a Distancia: de la práctica educativa hacia la inclusión sociocultural. Costa Rica.

López, M.; Espinoza A.; Flores. K. (2006). Percepción sobre las tecnologías de la información y la comunicación en los docentes de una universidad mexicana: el Centro Universitario del Sur de la Universidad de Guadalajara. Revista Electrónica de Investigación Educativa (REDIE), (1), 8.

Ministerio del poder popular para la educación universitaria. (s/f). Programa Fomento a la Educación Superior. [en línea] Disponible en: http://www.opsu. gob.ve/extranet/educacion distancia (consulta 2011, 3 de agosto).

Pérez, A. (2008). Presentación de SEDUCLA ante el consejo Universitario. Universidad Centroccidental Lisandro Alvarado.
Santos R. (2007). Implantación de las TIC en la educación y capacitación docente. [en línea] Disponible en: http://www.uca. edu.sv/deptos/letras/enplural/archivo/ a 2n1/articulos/arto1.htm \#autor (consulta 2011, 17 de septiembre).

Siemens, G. (2007). Conectivismo: Una teoría de aprendizaje para la era digital. [en línea] Disponible en: http://www.diegoleal.org/docs/2007/ Siemens(2004)-Conectivismo.doc (consulta 2011, 17 de septiembre).

Silvio, J. (2004). El aprendizaje mixto en la educación permanente: bases para una estrategia sistemática. Virtual Educa Barcelona.

Tobón, M. (2007). Diseño Instruccional en un entorno de aprendizaje abierto. Universidad Tecnológica de Pereira.

UNESCO. (1998). Declaración mundial sobre la educación superior en el siglo XXI: Visión y acción. [en línea] Disponible en: http://www.unesco.org/ education/educprog/wche/declaration spa.htm\#declaracion (consulta 2011, 4 de agosto).

UNESCO. (2008). Estándares de competencias en TIC para docentes. [en línea] Disponible en: http://www.eduteka.org/pdfdir/ UNESCOEstandaresDocentes.pd (consulta 2011, 2 de agosto).

Universidad Centroccidental Lisandro Alvarado. (UCLA) (2005). Directrices paralaformulación delperfilprofesional bajo el enfoque por competencias. Vicerrectorado Académico, Comisión Central de Currículo. Barquisimeto, Venezuela. 


\section{PERFIL ACADÉMICO Y PROFESIONAL DE LAS AUTORAS}

Graciela Henríquez Gabante. Ingeniera en Informática (1989) Universidad Centroccidental LisandroAlvarado; Magister Scientiarum en Ingenería Industrial mención Gerencia (1998) Universidad Experimental Politécnica Antonio José de Sucre; Doctorado en Educación mención Tecnología Instruccional y Educación a Distancia (2006) NOVA SOUTHEASTERN UNIVERSITY - Fischler Graduate School of Education. Docente investigadora Asociado, dedicación exclusiva de la Universidad Centroccidental Lisandro Alvarado. Miembro del Sistema de Educación a Distancia SEDUCLA- Cs. de la Salud y de Evaluación Institucional. Ganadora de premios CONABA y PPI.

E-mail: hgraciel@yahoo.com

Eunice Elena Ugel Garrido. Técnica Superior en Informática (1989) Instituto de Tecnología Antonio José de Sucre; Licenciatura en Administración mención: Organización y Sistemas (2006) Universidad Nacional Experimental Simón Rodríguez. Auxiliar docente, tiempo completo de la Universidad Centroccidental Lisandro Alvarado. Participa activamente en las funciones de Investigación y Extensión, ejecutando proyectos los cuales ha presentado en Congresos, Jornadas, Talleres, entre otros.

E-mail: euniceu@hotmail.com

\section{DIRECCIÓN DE LAS AUTORAS:}

Decanato Ciencias de la Salud

Universidad Centroccidental Lisandro Alvarado, UCLA

Av. Libertador con Av. "Andrés Bello"

Al lado del Hospital Central "Antonio María Pineda"

Barquisimeto. Estado Lara.

Venezuela

Fecha de recepción del artículo: 19/05/11

Fecha de aceptación del artículo: 23//9/11

\section{Como citar este artículo:}

Henríquez Gabante, G.; Ugel Garrido, E. E. (2012). Migración de lo presencial a lo virtual en la asignatura introducción a la computación del programa de enfermería de la UCLA. RIED. Revista Iberoamericana de Educación a Distancia, volumen 15, $\mathrm{n}^{\circ}$ 1, pp. 127-142. 